\title{
Do Dogs See the Ponzo illusion?
}

\author{
Sarah-Elizabeth Byosiere*, Lynna C. Feng, Nicholas J. Rutter, Jessica K. Woodhead, \\ Philippe A. Chouinard, Tiffani J. Howell, and Pauleen C. Bennett
}

School of Psychology and Public Health, La Trobe University, Victoria, Australia.

*Corresponding author (Email: S.Byosiere@latrobe.edu.au)

Citation - Byosiere, S-E., Feng, L.C., Rutter, N.J., Woodhead, J.K., Chouinard, P.A., Howell, T.J., \& Bennett, P.C. (2017). Do dogs see the Ponzo illusion? Animal Behavior and Cognition, 4(4), 396 - 412.

https://dx.doi.org/10.26451/abc.04.04.01.2017

\begin{abstract}
While domestic dogs (Canis familiaris) play a large role in human daily lives, little is known about how they perceive the visual world. Recent research suggests that dogs may perceive certain visual illusions differently than humans. To further evaluate geometric illusion susceptibility, eight dogs were assessed on their susceptibility to the Ponzo illusion. Four experiments were conducted: 1) a presentation of the Ponzo illusion with target circles in a 'grid inducer context', 2) a re-test of Experiment 1 after additional training, 3) a presentation of the Ponzo illusion with target rectangles in a 'grid inducer context' and 4) a presentation of the Ponzo illusion with target circles in a 'converging lines context.' A one-sample t-test of the dogs' responses to the Ponzo stimuli in Experiment 1 demonstrated illusion susceptibility at the group level; however, no individual dog performed significantly above chance in binomial tests. In Experiments 2, 3, and 4, one-sample t-tests found no significant results at the group level, although one or more dogs did demonstrate a small but significant effect. Taken together, then, there was limited evidence for dogs' susceptibility to the Ponzo illusion in a two-choice discrimination paradigm. As most animals tested previously have demonstrated human-like susceptibility to the Ponzo illusion, these findings have implications for theoretical explanations. The divergence of results between dogs and humans/other animals suggest that mechanisms underlying perception of the Ponzo illusion may differ across species and that care should be taken when using visual paradigms to test dogs' cognitive skills.
\end{abstract}

Keywords - Ponzo illusion; Geometric illusion; Domestic dog; Perception; Two-choice discrimination

Visual perception represents interpretation of retinal information by the brain, which allows humans and non-human animals (hereafter animals) to perceive the external world and act upon it (Haber \& Hershenson, 1973). However, visual perception does not always reflect reality, given that the brain often adapts visual information from the retina to fit preconceptions (Gregory, 2015). Geometric illusions provide an opportunity to examine perceptual processes by inducing these largely adaptive preconceptions, or 'corrections' to visual information, in contexts where visual corrections are unnecessary.

One of the most widely studied visual illusions is the Ponzo illusion. This illusion typically consists of two equally sized targets (e.g., circles or lines) that appear unequal when superimposed over converging lines (Figure 1). Several explanations for the Ponzo illusion have been proposed. One of the most popular, Gregory's (1963) Inappropriate Constancy-Scaling theory proposes misapplication of size constancy arising from the brain's interpretation of the converging lines as depth cues (for review of multiple theories see Humphrey \& Morgan, 1965; Newman \& Newman, 1974; Sperandio \& Chouinard, 2015). Thus, the circle nearest to the apex of the converging lines appears larger as it is perceived as 
A)

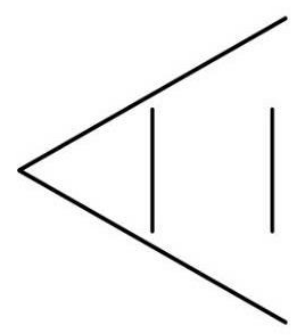

B)

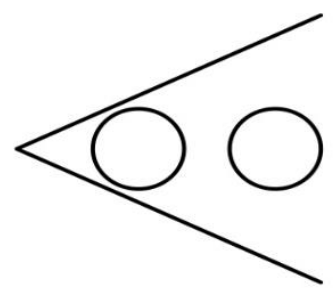

C)

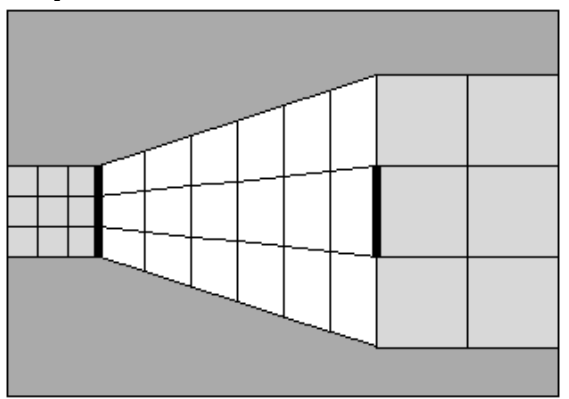

Figure 1. Three examples of the Ponzo illusion, where $\mathbf{A}$ and $\mathbf{C}$ utilize identically sized horizontal bars, and $\mathbf{B}$ utilizes identically sized circles. A and $\mathbf{B}$ are presentations using a 'converging lines' context whereas $\mathbf{C}$ is a presentation using a 'grid inducer' context. Here, the object closer to the apex of the converging lines, or in the smaller inducer grid, appears larger than the other object.

being further away, causing the viewer to inappropriately compensate for this distance in order to maintain size constancy. In contrast, Assimilation theory has been proposed by Pressey (1971) to explain the Ponzo illusion based on two main postulates, which are as follows:

(1) Whenever judgments are made of a series of magnitudes, the smaller magnitudes in that series will be overestimated and the larger magnitudes will be underestimated. (2) Other things being equal, a context which falls within the attentive field will be more effective than a context outside that field (Pressey, 1971, p. 172).

Here, the target closest to the apex is said to appear larger as it is closer to the contextual lines than the alternative target. Furthermore, visually estimating the differences in stimuli size narrows the observer's attentive field in a tunnel-vision like way, through which little of the surrounding context is considered, except that closest to the stimuli. Consequently, the stimulus closest to the context lines is 'assimilated,' thereby causing the perceiver to overestimate its size (Fujita, 1997).

Regardless of the specific mechanisms inducing the Ponzo illusion, research to date suggests that most humans are susceptible to the illusion. However, there is some evidence of cross-cultural differences in susceptibility (Brislin \& Keating, 1976; Segall, Campbell, \& Herskovits, 1963), in addition to the fact that susceptibility may be inversely correlated with the size of an individual's primary visual cortex (Song, Schwarzkopf, \& Rees, 2011). Furthermore, the Ponzo illusion has also been widely assessed across animal taxa (Feng, Chouinard, Howell, \& Bennett, 2016). For instance, pigeons (Fujita, Blough, \& Blough, 1991), Sprague-Dawley rats (Nakagawa, 2002), horses (Timney \& Keil, 1996), rhesus macaques (Bayne \& Davis, 1983; Fujita, 1997), baboons (Barbet \& Fagot, 2002), and chimpanzees (Fujita, 1997; Imura, Tomonaga, \& Yagi, 2008) have all been studied, with all species to date demonstrating susceptibility to the illusion in the same manner as humans.

Dogs represent a unique model for examining illusory susceptibility. However, to our knowledge their susceptibility to the Ponzo illusion has not yet been examined. Over the last 30,000 years, humans and dogs have evolved within similar ecological niches (Wang et al., 2013). Given this shared evolutionary development, it is plausible that certain evolutionary pressures, such as habitat, may have driven co-evolutionary processes (Geisler \& Diehl, 2002). While little research has been conducted on the extent of visual perceptive abilities in domestic dogs, a basic understanding of how dogs detect light, process colors, and perceive motion, is available (Miller \& Murphy, 1995). To our knowledge, however, only two studies have gauged susceptibility to geometrical illusions in dogs. These provided puzzling results. Byosiere et al. (2016) found that dogs misperceived the Ebbinghaus-Titchener illusory stimuli, but responded in a reversed manner compared to humans and most other mammals, indicating that the 
stimulus humans perceive as being larger is perceived by dogs as being smaller. Furthermore, while dogs as a group appear not to be susceptible to the Delboeuf illusion, certain individuals may demonstrate susceptibility, also in a reversed direction than humans and other mammals (Byosiere et al., 2016; Miletto Petrazzini, Bisazza, \& Agrillo, 2016).

Variation in illusion susceptibility is not unique to dogs. A variety of studies have found humanlike susceptibility, no susceptibility, or reversed susceptibility in animals (e.g., Agrillo, Parrish, \& Beran, 2014; Fujita, 1996, 1997; Murayama, Usui, Takeda, Kato, \& Maejima, 2012; Nakamura, Watanabe, \& Fujita, 2008; Sovrano, Albertazzi, \& Salva, 2014; Watanabe, Nakamura, \& Fujita, 2011, 2013). Additionally, even within species mixed evidence has been observed in regards to susceptibility to the same illusion (e.g., Nakamura et al., 2008; Nakamura, Watanabe, \& Fujita, 2014; Salva, Rugani, Cavazzana, Regolin, \& Vallortigara, 2013). Therefore, the intriguing findings observed in previous studies of dogs warrant further investigation of dogs' illusory susceptibility.

The aim of this study was to determine whether dogs demonstrate susceptibility to the Ponzo illusion. We predicted that dogs would accurately indicate the relative size of two differently sized target stimuli, following an appropriate training regimen, regardless of the background context in which these stimuli were presented. However, we had no a-priori predictions in regards to performance in the illusion condition. While susceptibility to the Ponzo illusion is widespread and has been observed in humans and other mammals (Barbet \& Fagot, 2002; Bayne \& Davis, 1983; Fujita, 1996, 1997; Fujita et al., 1991; Imura et al., 2008; Nakagawa, 2002; Timney \& Keil, 1996), the fact that dogs have previously demonstrated reversed susceptibility or no susceptibility when presented with other geometric illusions (Byosiere et al., 2016; Miletto Petrazzini et al., 2016) meant that we were unable to predict if dogs would perceive the illusion at all and, if they did, whether this would be in the same or opposite manner to humans.

\section{General Methods}

\section{Subjects}

Eight companion dogs (Canis familiaris), six females and two males, participated in this study (at the start of Experiment 1 , mean age $=1$ year, 8 months; age range $=9$ months to 6.5 years). All dogs were purebred Lagotto Romagnolos and sexually intact except for two (Gabbie and Baxter) who were spayed or neutered respectively during the period of data collection. All dogs had previously participated in an experiment assessing illusion susceptibility (Byosiere et al., 2016) as well as one testing relational class concepts (Byosiere et al., under review).

\section{Apparatus}

Testing was conducted in the Canine Nose-Touch Testing Apparatus, a $1540 \mathrm{~mm}$ x $600 \mathrm{~mm}$ x $600 \mathrm{~mm}$ wooden apparatus designed for a different study (Byosiere et al., 2016), in order to reduce experimenter-expectancy effects and environmental distractions. The testing chamber was equipped with a $508 \mathrm{~mm}$ Dell® widescreen computer monitor for stimulus presentation $(1280 \mathrm{x} 800$ pixels; one pixel measures approximately $0.3 \mathrm{~mm}^{2}$ ), positioned with the lower edge approximately $24 \mathrm{~cm}$ above ground level. Below it, at ground level, was a remotely controlled treat dispenser (Treat \& Train ${ }^{\circledR}$ ) that, when activated, distributed one piece of semi-moist dry dog food (Nature's Gift巴 Mini Kangaroo, or Good-o® treats). A video camera was mounted above the monitor to record each trial. Data acquisition was conducted through customized programs developed specifically for this study by the research team using Processing 2.2.1 on a Dell Latitude D531 LCD laptop.

\section{Training Procedure}

Dogs were initially trained based on their individual performance, beginning in April 2015 and continuing for four months, to target a black $80-\mathrm{mm}$ diameter circle via a nose-touch. Target stimuli were 
first presented in a trainer's hand, and then glued to a wall $(45 \mathrm{~cm}$ above the ground). This behavior was transferred to nose-touching a 62-mm circle presented in random locations on a computer monitor housed in an open room. Once this stimulus training was complete, the dogs were familiarized with the Canine Nose-Touch Testing Apparatus and the task was generalized to multiple stimulus pairs, each comprising a small and large circle. All dogs were trained to select the relatively larger circle, except for one (Baxter), who was trained to select the smaller circle. This is due to the fact that the dogs were originally randomly assigned into two groups, Audrey, Gabbie, Hamish, and Molly being trained to select the larger circle and Baxter, Beth, Eliza, and Lulu being trained to select the smaller one. Each dog was trained for 12 weeks, following which he or she was tested for accuracy. Despite previous reports that dogs do not show an innate preference for large over small stimuli (Tapp et al., 2004), only one dog in the small circle group, Baxter, met the criteria of $80 \%$ correct responses. All four dogs in the large circle group met the same criteria. We decided that rather than exclude Beth, Eliza and Lulu from the study, we would retrain them to select the large circle. When tested four weeks later, all three met the criteria of $80 \%$ correct responses.

At the conclusion of the pre-training process, all dogs could reliably discriminate between two identical circle stimuli varying in pixel diameter size by 60\% (106 pixels, $31.8 \mathrm{~mm}$, and 207 pixels, 62.1 $\mathrm{mm}$ in diameter; 207 pixels, $62.1 \mathrm{~mm}$, and 403 pixels, $120.9 \mathrm{~mm}$ in diameter) averaging an accuracy rate of $78.4 \%(65-87 \%)$. When presented with two circles that were identical in size (207 pixels, $62.1 \mathrm{~mm}$, in diameter), each dog's performance was at chance levels of $54.7 \%(47.5-72 \%)$. The dogs typically participated in the experiments enthusiastically. Although data were recorded manually, the dog's responses were typically very easy to distinguish. In our previous study, we assessed inter-observer reliability using a random sample of the video-recorded data $(n=150)$ and found it was $99.3 \%$ (Byosiere et al., 2016).

\section{Testing Procedure}

Two experimenters (Exp A, Exp B) were present at all times during testing. Exp A sat to the left of the Canine Nose-Touch Testing Apparatus and controlled a laptop computer. This person presented and removed the stimuli, while recording the data. Exp B was positioned at the front-right side of the apparatus, out of sight from the dog. The trial began when the stimuli were presented on the screen and the dog was positioned at the entrance of the apparatus. Once in the apparatus and unable to see either experimenter, the dog moved to the screen and selected one stimulus by nose-touching the image. If the dog chose correctly, Exp B, watching the dog through the top of the apparatus, activated the remotely controlled treat dispenser located below the computer monitor and signaled to Exp A to record a correct choice. If the dog chose incorrectly, and Exp B relayed a signal to Exp A to remove the stimuli and thereby end the trial. Exp A then called the dog to return to the entrance of the apparatus to resume the starting position for the next trial. Once the dog returned to Exp A, the dog received occasional food rewards. This meant that the dogs were rewarded simply for participating in the experiment, which helped to maintain motivation even when performance was suboptimal.

Before each experiment, all dogs underwent familiarization sessions in which the target stimuli were presented over a non-illusory background (Figure 2) to acquaint the dogs with new images, such as inducers and/or colors, which would be present in the experimental trials. In these sessions, target stimulus pairs were presented in blocks of ten trials, with five presentations each of $S_{4}-S_{7}$ and $S_{7}-S_{10}$ stimuli being included in random order. The correct stimulus pseudo-randomly appeared on each side for an equal number of times in accordance with randomly selected Gellerman (1933) sequences. Six types of familiarization trials were conducted for Experiments 1 and 3, and one type for Experiments 2 and 4 (Figure 2). Type 6 was the only familiarization type conducted for Experiment 2 as this was a re-test of Experiment 1. Dogs progressed to the next type of familiarization trial only after reaching a criterion of $>$ $90 \%$ correct in a single ten-trial block, or $>70 \%$ correct across each of two consecutive blocks. In order to continue onto testing sessions, the dogs were required to successfully complete all familiarization sessions. In any case where a dog did not meet the criteria after seven attempts, the dog was excluded from participating in the testing condition. 


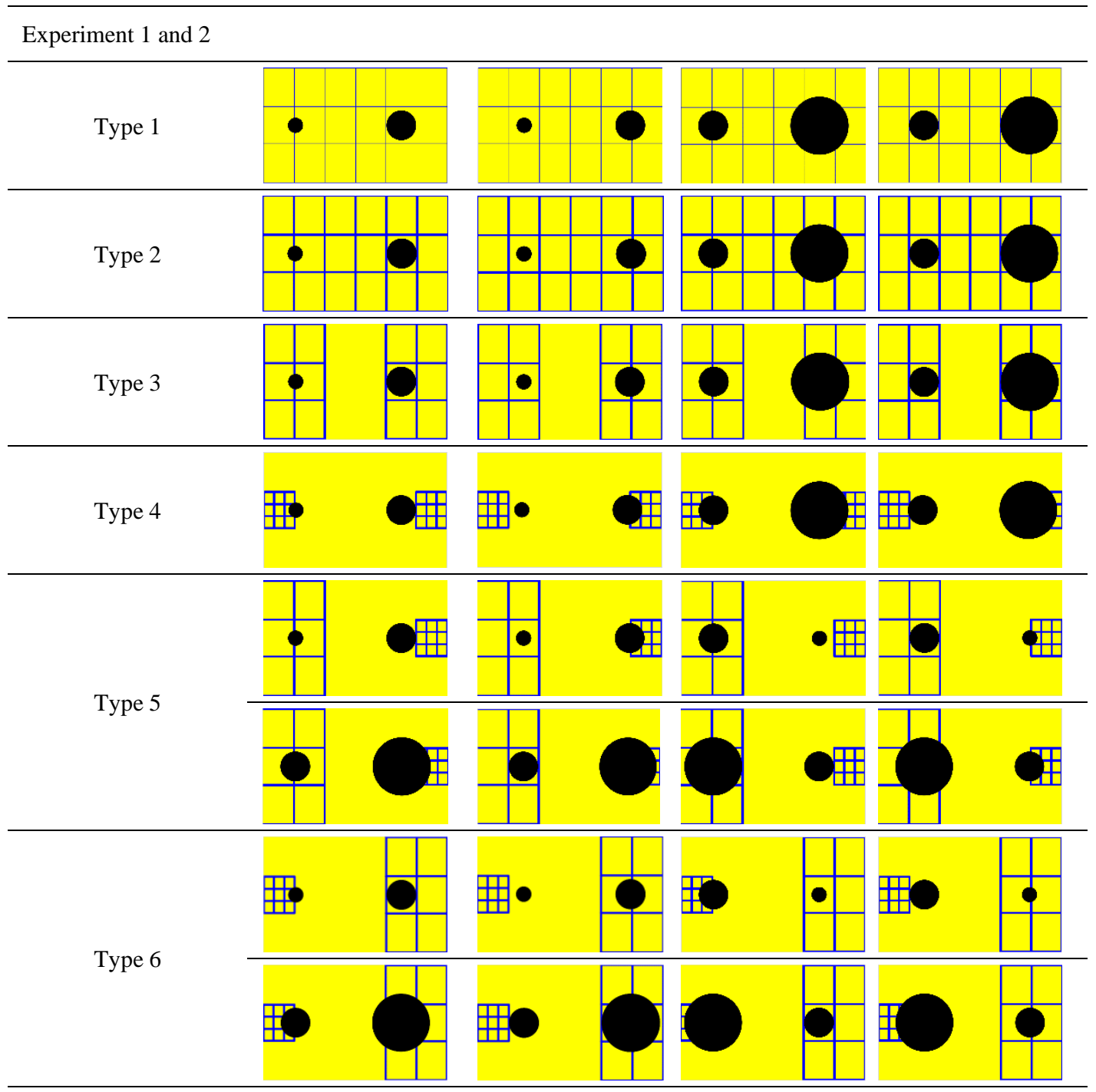

Experiment 3

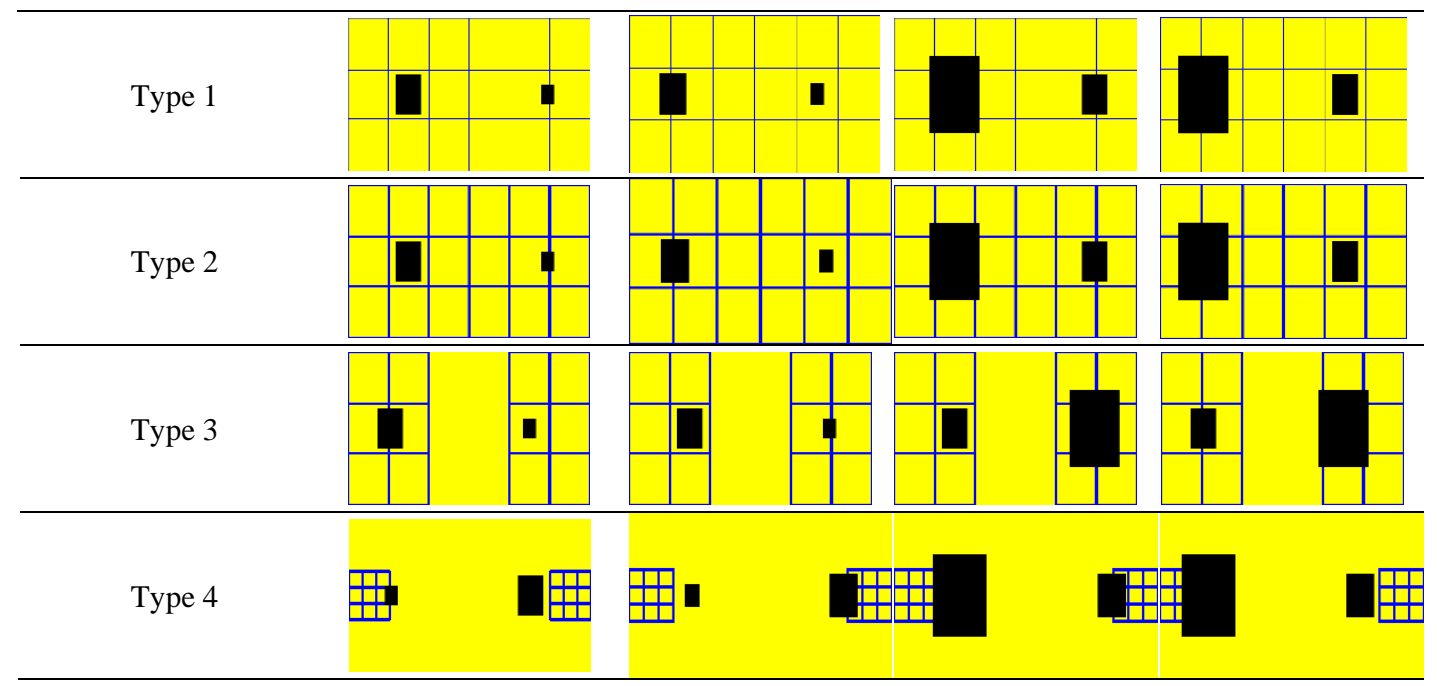




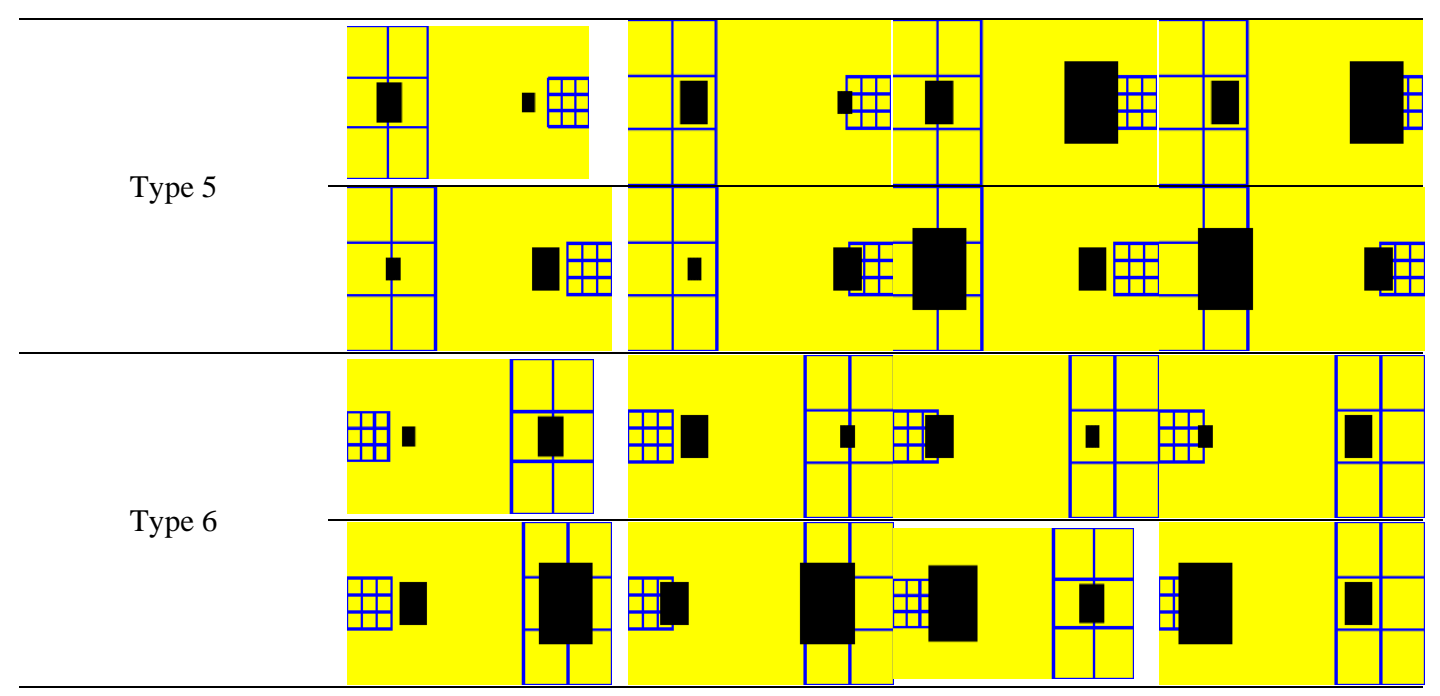

Experiment 4

Type 1
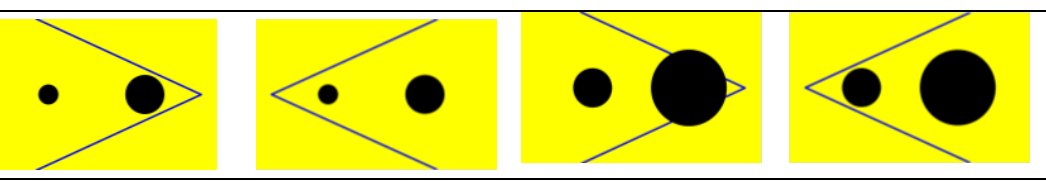

Figure 2. Familiarization stimuli used in Experiment 1 and 2 employing the circle 'grid inducer context', Experiment 3 employing the rectangle 'grid inducer context', and Experiment 4 employing the 'converging lines context.'

In the test sessions, stimulus pairs were presented in three sets: $S_{4}-S_{7}$ and $S_{7}-S_{10}$ represented control conditions, and $\mathrm{S}_{7}-\mathrm{S}_{7}$ represented experimental trials. Ten blocks of ten trials were administered for each experiment, resulting in 100 trials per dog (60 control trials, 40 experimental trials). Each round of ten trials consisted of six control trials, including three comparisons of $\mathrm{S}_{4}-\mathrm{S}_{7}$ and three comparisons of $\mathrm{S}_{7}-\mathrm{S}_{10}$ (control condition), and four experimental trials of $\mathrm{S}_{7}-\mathrm{S}_{7}$ comparisons (illusion condition).

A response in the illusion condition was considered correct when the target stimulus typically seen as 'perceptually larger' to humans was chosen. However, so as not to reinforce any particular way of responding, reinforcement for the experimental trials was pseudo-randomized using a Gellerman sequence to order the trials. If the Gellerman sequence dictated that the target stimulus on the left for a particular experimental trial should be correct, it was deemed thus for the purposes of reinforcement. This was the case even though the target stimuli were identical in size, and even though a particular target stimulus may have been 'correct' insofar as humans perceived it as perceptually larger. As Baxter was the only subject trained to select the smaller target stimulus, his results indicate which stimulus he perceived as smaller and are notated in the data presented below.

\section{Compliance with Ethical Standards}

All applicable international, national, and/or institutional guidelines for the care and use of animals were followed. All procedures performed in the following experiments were in accordance with the ethical standards of La Trobe University Animal Ethics Committee (approval number: AEC15-18 and AEC16-26). This article does not contain any studies with human participants performed by any of the authors. Informed consent was obtained from all dog owners included in the study.

\section{Experiment 1}

Experiment 1 was conducted to determine whether dogs demonstrated susceptibility to the Ponzo illusion. Target stimuli consisted of black (RGB values $0,0,0$; Luminance $0.18 \mathrm{c} / \mathrm{m}^{2}$ ) circles presented on a highly contrasting yellow background (RGB values 255, 255, 0; Luminance $163.6 \mathrm{c} / \mathrm{m}^{2}$ ) with blue 
contextual grids (RGB values 0, 0, 255; Luminance $16.05 \mathrm{c} / \mathrm{m}^{2}$ ) (Figure 3). The colors used were determined based on the fact that dogs are dichromatic, with two types of cone photoreceptor cells, for blue and yellow (Jacobs, Deegan, Crognale, \& Fenwick, 1993; Neitz, Geist, \& Jacobs, 1989) and based on previous research suggesting dogs are capable of perceiving differences between yellow and blue stimuli (Kasparson, Badridze, \& Maximov, 2013).
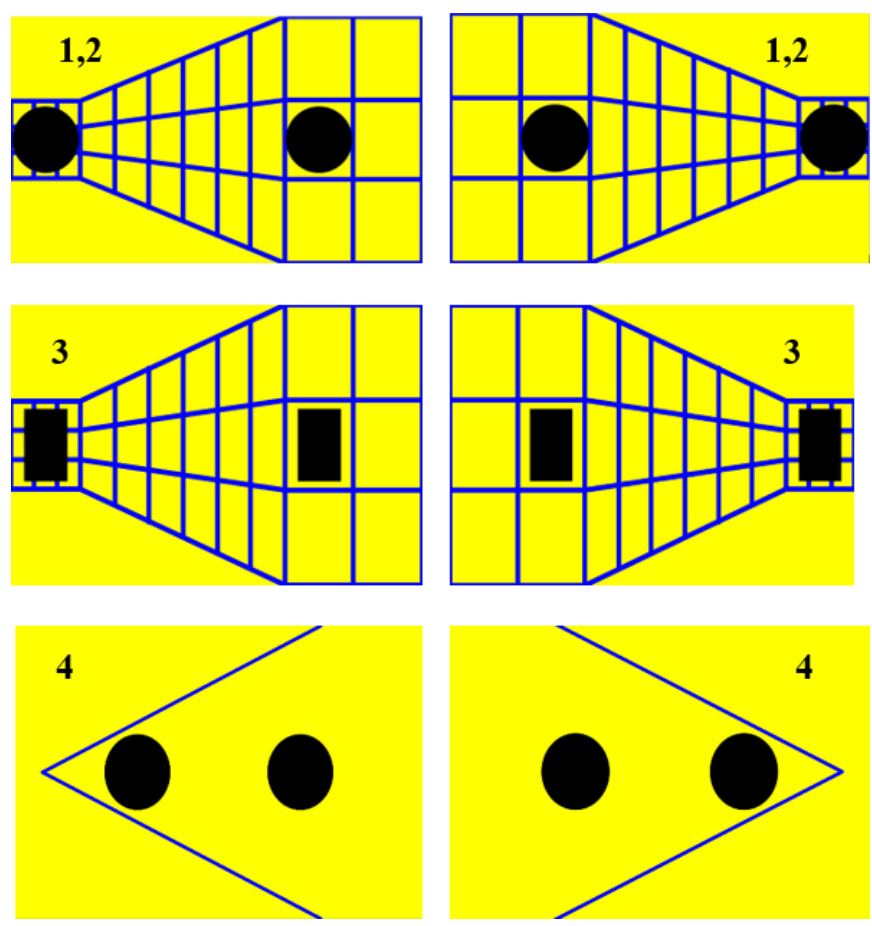

Figure 3. Ponzo illusion used in Experiments 1, 2, and 3 employing the 'grid inducer context' and the classic presentation in Experiment 4 employing the 'converging lines context.' The perceptually larger target stimulus appears on the left in the image on the left, and on the right in the image on the right.

The circles sizes were based on an array of 12 circles used previously to test transpositions in pigeons (Lazareva, Miner, Wasserman, \& Young, 2008; Lazareva, Wasserman, \& Young, 2005; Lazareva, Young, \& Wasserman, 2014) and in this same group of dogs (Byosiere et al., 2016). Three circle stimuli were used: $S_{4}$ circles were 106 pixels or $31.8 \mathrm{~mm}$ in diameter; $S_{7}$ circles were 207 pixels or $62.1 \mathrm{~mm}$ in diameter; $\mathrm{S}_{10}$ stimuli were 403 pixels or $120.9 \mathrm{~mm}$ in diameter (Figure 4). The circles were presented in pairs of $S_{4} S_{7}$ and $S_{7-} S_{10}$, to ensure that the dogs were not continually reinforced for choosing a single stimulus.

\section{Experiment 2}

Due to the significant group effect observed in Experiment 1, but the lack of an individual effect, Experiment 2 was conducted to replicate Experiment 1. Therefore, the stimuli and procedure were identical to those listed above. 
$\mathrm{S}_{4}$

Pixels
$\mathrm{S}_{7}$

Diameter 207
$\mathrm{S}_{10}$

Diameter: 403
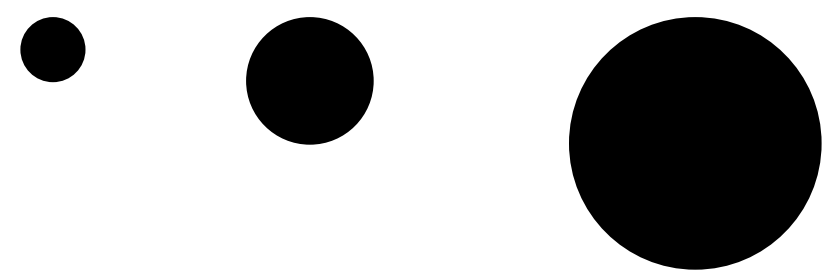

Figure 4. Schematic representation of circle stimuli implemented in the study with pixel values representing the diameter. Circles are provided to aid conceptual understanding only and are not actual size.

\section{Experiment 3}

In Experiment 3, we aimed to clarify the findings observed in Experiments 1 and 2. To determine if the results of the replication experiment (Experiment 2) were due to familiarity with the stimuli, we adapted the experiment and used novel target stimuli. Experiment 3 was identical to Experiments 1 and 2, barring the fact that rectangles were used as target stimuli instead of circles (Figure 5). Target stimuli consisted of black (RGB values 0,0,0; Luminance $0.18 \mathrm{c} / \mathrm{m}^{2}$ ) rectangles presented on a highly contrasting yellow background (RGB values 255, 255, 0; Luminance $163.6 \mathrm{c} / \mathrm{m}^{2}$ ) with blue contextual grids (RGB values 0, 0, 255; Luminance $16.05 \mathrm{c} / \mathrm{m}^{2}$ ) (Figure 3). Rectangle stimuli were chosen based on previous research conducted in our lab that indicated successful generalization from circles to novel rectangle shapes by the dogs in this study (Byosiere et al., under review). Three rectangle stimuli were presented in pairs of approximately $S_{4} S_{7}$ and $S_{7} S_{10} . S_{4}$ rectangles were 68.9 pixels in width and 106 pixels in height $(20.6 \times 31.8 \mathrm{~mm}) . \mathrm{S}_{7}$ rectangles were 134.5 pixels in width and 207 pixels in height $(40.3$ $\mathrm{x} 62.1 \mathrm{~mm}) . \mathrm{S}_{10}$ rectangles were 261.9 pixels in width and 403 pixels in height $(478.5 \mathrm{x} 120.9 \mathrm{~mm})$.

$\mathrm{S}_{4}$

Pixels

Width: 68.9

Height: 106

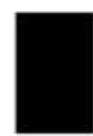

$\mathrm{S}_{7}$

Width: 134.5

Height: 207

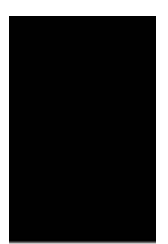

$\mathrm{S}_{10}$

Width: 261.9

Height: 403

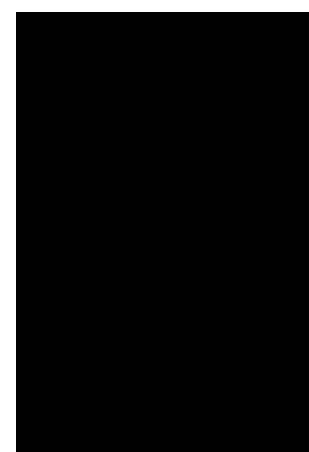

Figure 5. Schematic representation of rectangle stimuli implemented in the study with pixel values representing the width and height. Rectangles are provided to aid conceptual understanding only and are not actual size. 


\section{Experiment 4}

Due to the differences observed in the results across Experiments 1, 2, and 3, we aimed to verify if these findings were due to the context in which the stimuli were presented. Therefore, in Experiment 4 we utilized the classic presentation in which the Ponzo illusion is usually presented. Target stimuli once again consisted of black (RGB values $0,0,0$; Luminance $0.18 \mathrm{c} / \mathrm{m}^{2}$ ) circles presented on a highly contrasting yellow background (RGB values 255, 255, 0; Luminance $163.6 \mathrm{c} / \mathrm{m}^{2}$ ) with blue converging lines (RGB values 0, 0, 255; Luminance $16.05 \mathrm{c} / \mathrm{m}^{2}$ ) (Figure 3).

\section{Data Analysis}

To determine if dogs were performing significantly above chance, two-tailed binomial tests were conducted on each dog's individual responses in the control conditions and the illusion condition. Alpha was set at 0.05 and chance levels of performance were considered to be 0.5. Effect sizes were calculated for each illusion condition based on the means of the group. To examine group performances each illusion was analyzed using a two-tailed, one-sample $t$-test, conducted on the dogs' average proportion correct. Effect sizes (Cohen's $d$ ) are also reported.

\section{Results}

\section{Experiment 1}

The dogs generally performed extremely well in the familiarization sessions. On average, it took 1.27 blocks of ten trials for the group as a whole to progress to the next level, with stimulus types Two and Six appearing to be the most difficult, averaging 1.38 blocks each.

Figure 6 shows individual performance data for each dog in the test sessions. It is evident that performance in the control conditions was uniformly high, as the average level of performance was $82.7 \%$ \pm 6.7 . Binomial tests indicated that all dogs were significantly above the chance score of $50 \%$. In contrast,

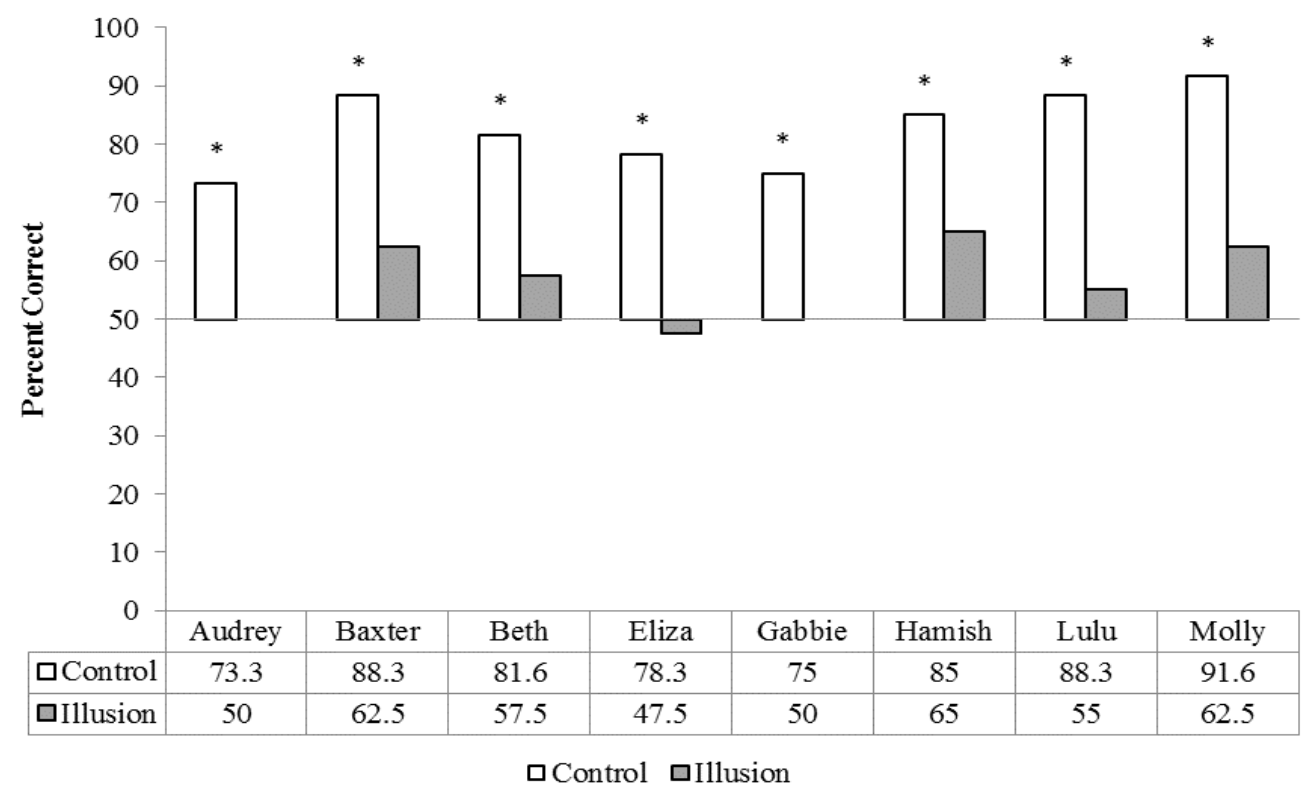

Figure 6. Individual percent correct for control and illusion conditions in Experiment 1.

*Indicates that the binomial result significantly differed from chance level at $p<0.05$. Correct trials in the illusion condition were defined as those in which the dog selected the stimulus that, to humans, would appear larger (or smaller in the case of Baxter). 
dogs averaged $56.25 \% \pm 6.7$ in the illusion condition. Despite this, the result of the two-tailed one-sample $t$-test conducted on the dogs' average proportion correct for the illusion condition in Experiment 1 was significant, indicating susceptibility to the illusion in a human-like direction $[t(7)=2.65, p=0.033, S D=$ 6.68, Cohen's $d=0.9328$ ]. While most dogs, individually, showed a slight susceptibility to the illusion, in that their score was above, rather than below, the chance level performance of $50 \%$ correct, there was insufficient evidence to suggest that any individual dog was susceptible to the illusion above chance levels at $\alpha=0.05$ ( $p \geq 0.06$ for all tests). Interestingly, the effect size was extremely large considering dogs averaged only $56.25 \%$ and no individual performed significantly above chance. This likely reflects the small standard deviation observed across trials.

\section{Experiment 2}

Given that, as a group, the dogs demonstrated significant susceptibility to the Ponzo illusion in Experiment 1, while no dog performed significantly above chance individually, it was decided to repeat the experiment. Unfortunately, in the intervening period the dogs had been used in an additional study testing their sensitivity to size differences of various magnitudes (Byosiere et al., in prep). They were therefore extremely practiced at the discrimination task. Regardless, the dogs performed extremely well in the repeat presentation of familiarization Type 6. On average, it took 1.25 blocks of ten trials for the group as a whole to progress from the single familiarization session to the test session.

Figure 7 shows individual performance data for each dog. Performance in the control conditions was again uniformly high, as the average level of performance was $94.7 \% \pm 6.9$. Binomial tests indicated that all dogs were significantly above the chance score of $50 \%$. In contrast, dogs as a group averaged only $48.1 \% \pm 12.5$ in the illusion condition. Results of the two-tailed, one-sample $t$-test for the illusion condition of the group in Experiment 2 were not significant $[t(7)=0.42, p=0.685, S D=12.52$, Cohen's $d=-0.1498]$. Only one dog, Baxter, performed significantly above chance level, demonstrating humanlike susceptibility to the illusion $(72.5 \%, p<0.01)$. All other dogs recorded responses that did not significantly differ from chance levels $(p \geq 0.11)$.

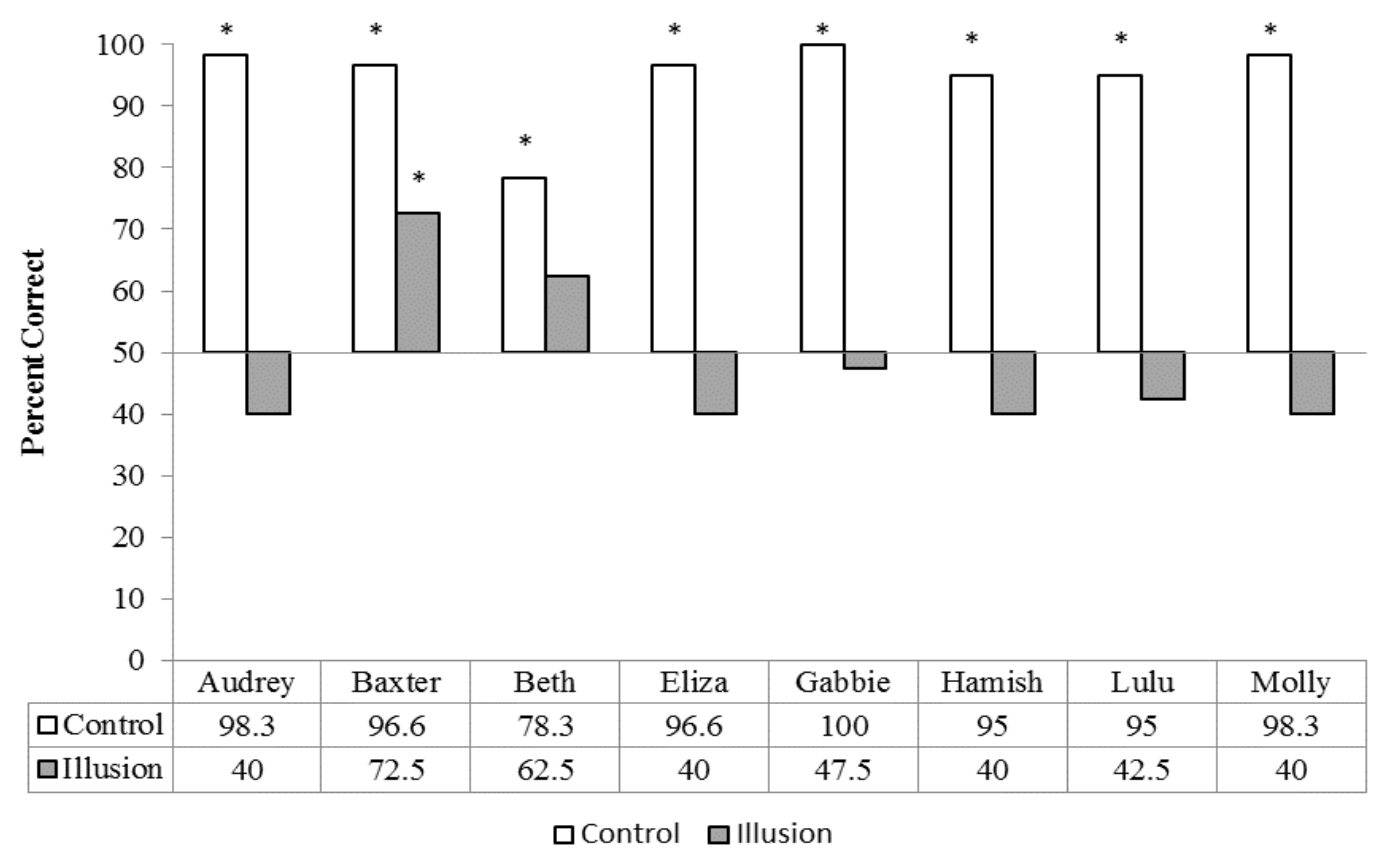

Figure 7. Individual percent correct for control and illusion conditions in Experiment 2.

*Indicates that the binomial result significantly differed from chance level at $p<0.05$. Correct trials in the illusion condition were defined as those in which the dog selected the stimulus that, to humans, would appear larger (or smaller in the case of Baxter). 


\section{Experiment 3}

Given the differences observed in the results of Experiment 1 and 2, we conducted Experiment 3 in order to present the dogs with a novel target stimulus, rectangles. Once again, the dogs performed extremely well in the familiarization sessions. On average, it took 1.81 blocks of ten trials for the group as a whole to progress to the next level, with Type 1, 3, 4 and 6 stimuli (see Figure 2) being the most difficult and requiring an average of 2 blocks of ten trials. Performance between dogs was more variable in this experiment, however, with Beth and Eliza being excluded from testing as they reached the maximum seven attempts in Type 1 and 4 , respectively.

Figure 8 shows individual performance data for each dog. Performance in the control conditions was once again uniformly high, as the average level of performance was $85 \% \pm 7$. Binomial tests indicated that all dogs were significantly above the chance score of $50 \%$. In contrast, dogs as a group averaged only $45.8 \% \pm 15.5$ in the illusion condition. Results of the two-tailed, one-sample $t$-test for the illusion condition of the group in Experiment 3 were not significant $[t(5)=0.66, p=0.53, S D=15.46$, Cohen's $d=-0.269$ ]. Again, individuals varied greatly in the illusion condition. Consistent with his performance in Experiment 2, Baxter performed significantly above chance level, indicating susceptibility to the illusion in the same direction as humans $(p=0.03)$. Gabbie and Lulu performed significantly below chance level, indicating susceptibility in the opposite direction to humans $(p \leq 0.03)$. The three remaining dogs performed at chance $(p \geq 0.21)$.

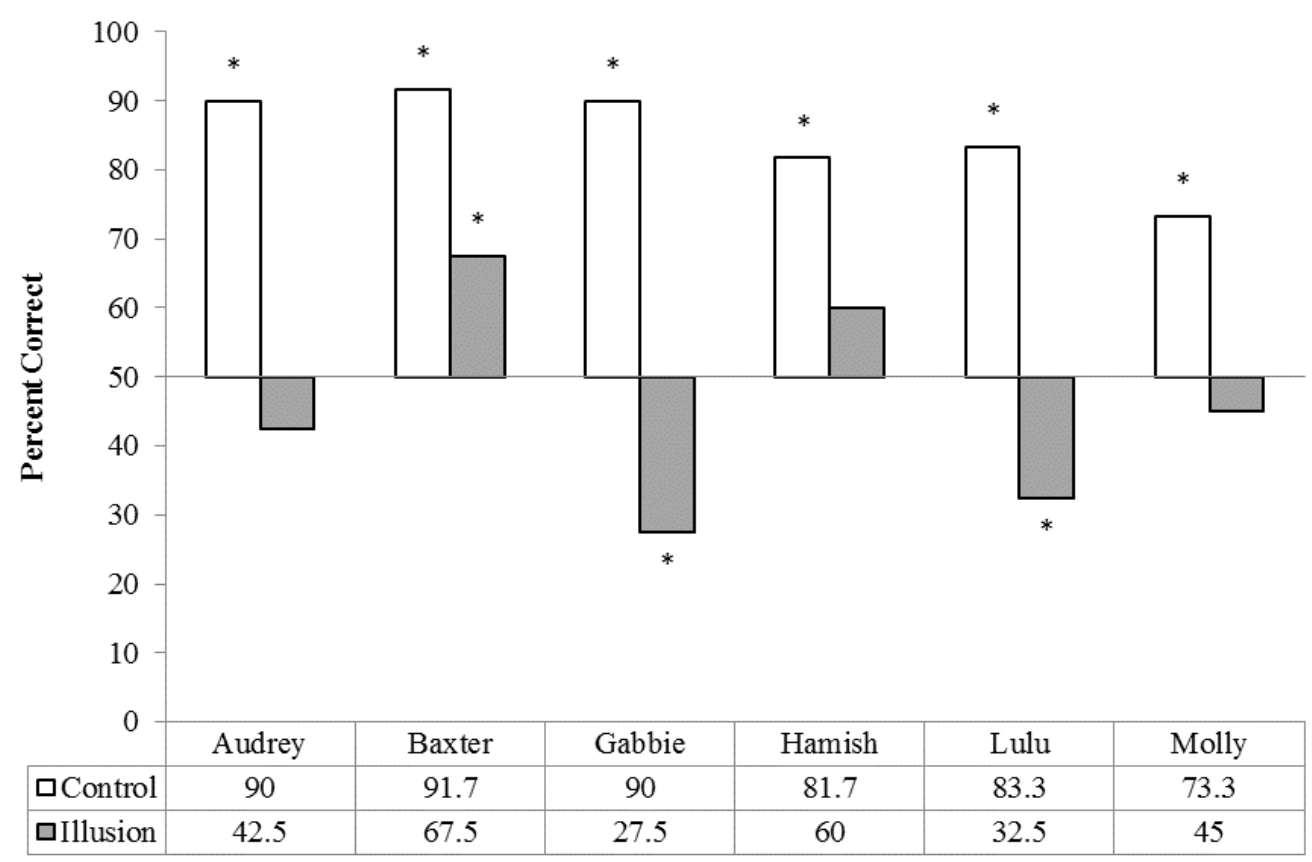

Figure 8. Individual percent correct for control and illusion conditions in Experiment 3.

*Indicates that the binomial result significantly differed from chance level at $p<0.05$. Correct trials in the illusion condition were defined as those in which the dog selected the stimulus that, to humans, would appear larger (or smaller in the case of Baxter).

\section{Experiment 4}

In order to assess illusion susceptibility in a commonly presented Ponzo context, we conducted Experiment 4. Once again, dog performed extremely well in the familiarization sessions. All dogs performed at $90 \%$ or above (average of $97.14 \%$ ) and therefore passed through to the illusion condition. Lulu was excluded from participating in this experiment, as she was pregnant at the time of testing. 
Figure 9 shows individual performance data for each dog. Performance in the control conditions was once again uniformly high, as the average level of performance was $95.2 \% \pm 4.7$. Binomial tests indicated that all dogs were significantly above the chance score of $50 \%$. In contrast, dogs as a group averaged only $56.4 \% \pm 18.6$ in the illusion condition. Results of the two-tailed one-sample $t$-test for the illusion condition of the group in Experiment 4 were not significant $[t(6)=0.95, p=0.37, S D=18.64$, Cohen's $d=0.34]$. Performance in the illusion condition was similar to the previous three experiments. Baxter and Gabbie performed significantly above chance level, indicating illusion susceptibility in the same direction as humans $(p \leq 0.01)$. The remaining five dogs performed at chance levels $(p \geq 0.06)$.

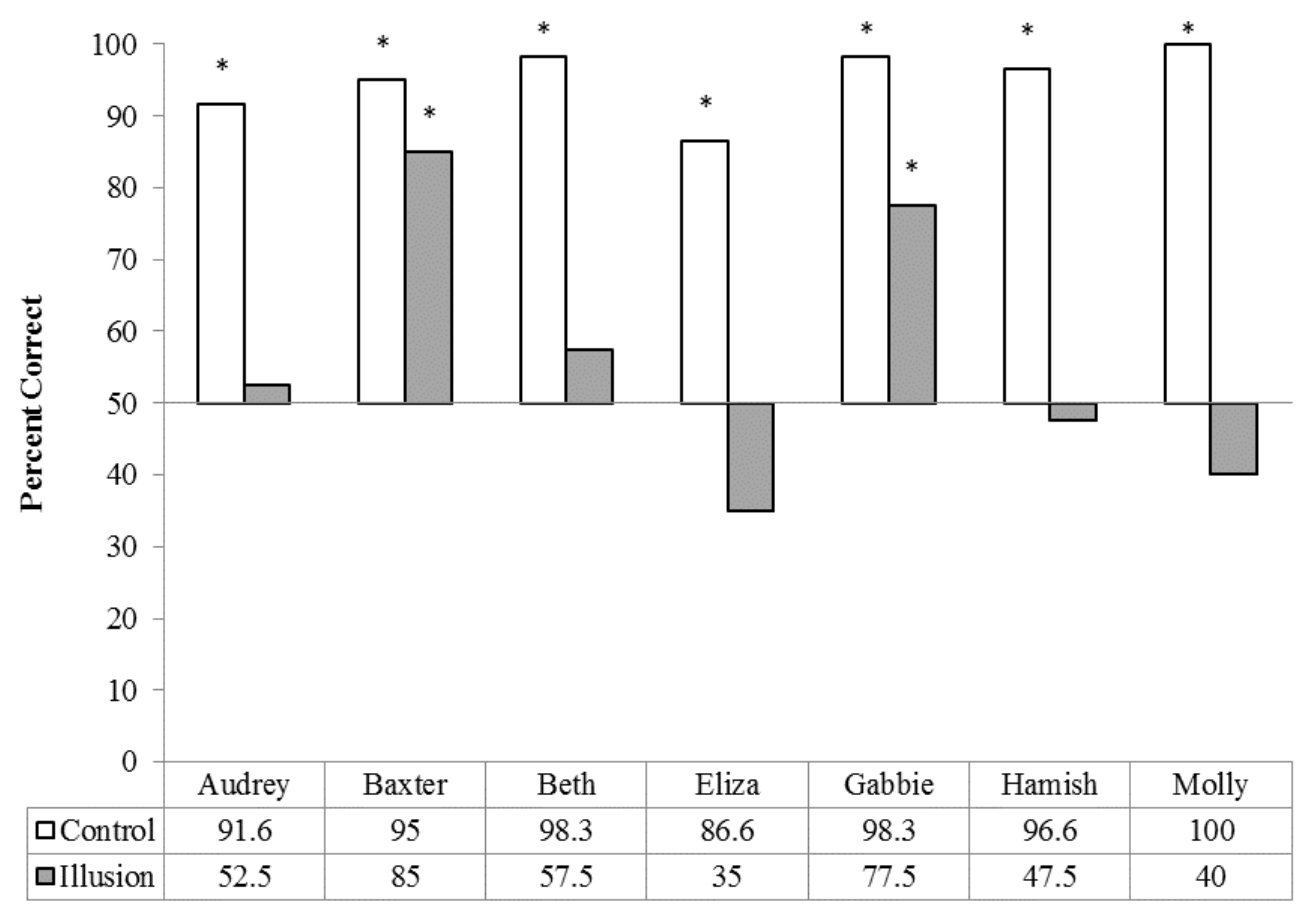

Figure 9. Individual percent correct for control and illusion conditions in Experiment 4.

*Indicates that the binomial result significantly differed from chance level at $p<0.05$. Correct trials in the illusion condition were defined as those in which the dog selected the stimulus that, to humans, would appear larger (or smaller in the case of Baxter).

\section{Summary of Results}

To summarize group performance across Experiments 1-4, the dogs generally performed at or around chance when presented with the varying presentations of the Ponzo illusion. Dogs only performed significantly above chance, as a group, when presented with the circle 'grid inducer' context in Experiment 1, demonstrating (weak) susceptibility in the same direction as humans $(p=0.033$ ) (Figure 10). Dogs performed at chance as a group across Experiments $2(p=0.685), 3(p=0.53)$, and $4(p=0.37)$ (Figure 10). There were exceptions in each experiment, however. While some dogs performed significantly above chance in either both a human-like and reversed direction, only one dog, Baxter, showed a weak but consistent tendency towards human-like susceptibility. These instances are discussed in more detail below. 


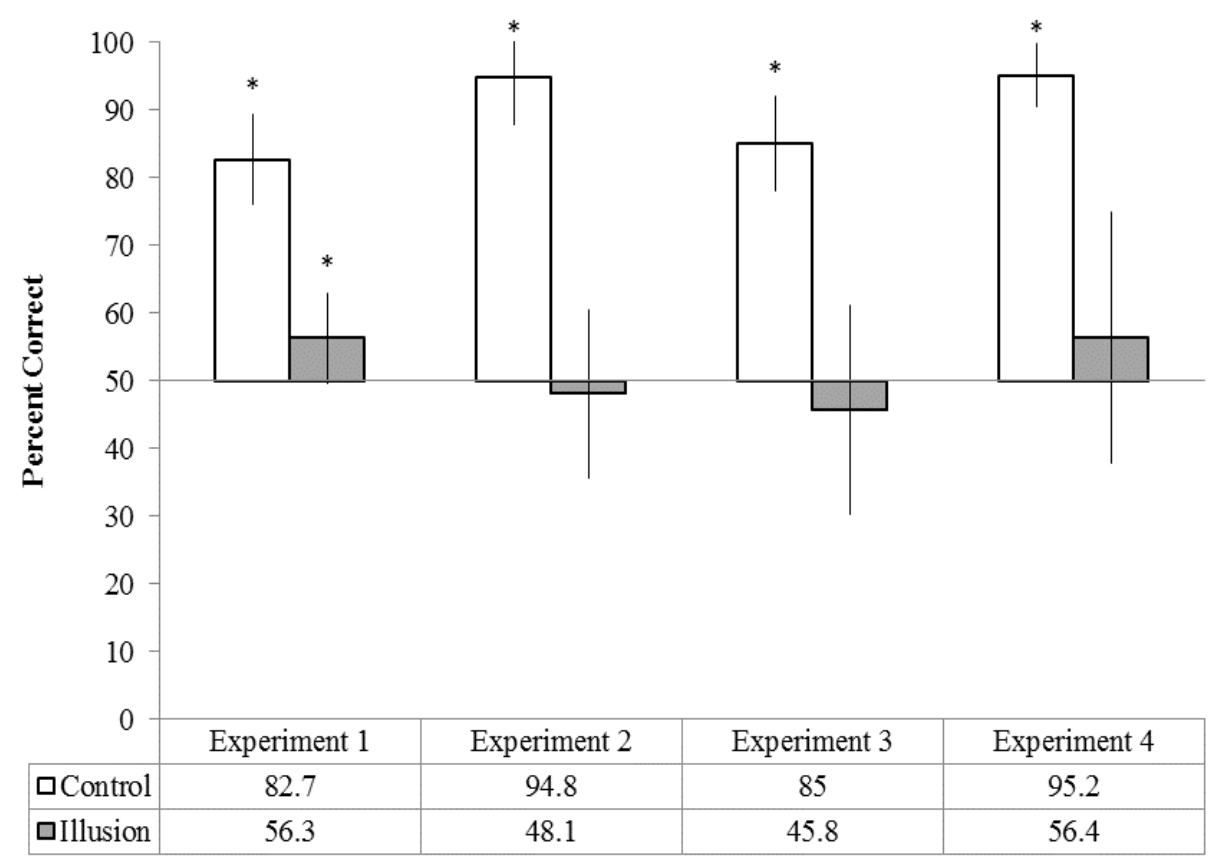

Figure 10. Percent of correct choices at the group level for the control and illusion condition for Experiments 1, 2, 3, and 4, with group standard deviations for each experiment.

*Indicates that the t-test at the group level, comparing correct responses against chance, was significant at $p<0.05$. Error bars indicate standard deviation.

\section{Discussion}

The current study examined susceptibility to the Ponzo illusion in dogs across a variety of presentations, utilizing both 'grid inducer' and 'converging lines' contexts. While the $t$-test on the group data for Experiment 1 was suggestive that the dogs were responding to illusory stimuli significantly above chance and in the same direction as humans and other animals, no individual dog performed significantly above chance. However, when the exact same experiment was run again, as Experiment 2, no significant group result was found. The results of Experiments 3 and 4 also indicated that the dogs, as a group, were responding to illusory stimuli at chance. Taken together, there was a lack of compelling evidence for discernible susceptibility to the Ponzo illusion in a two-choice discrimination paradigm when utilizing both 'grid inducer' and 'converging lines' contexts.

Despite this overall conclusion, a number of anomalies indicate that additional testing may be required before it can be concluded with certainty that dogs are not susceptible to the Ponzo illusion. First, the dogs, as a group, did perform significantly above chance in Experiment 1, even though no single individual dog demonstrated performance significantly above chance. This result can be explained by the fact that most dogs were trending just above chance performance (at approximately 56\%). Additionally, the standard deviation for the dogs as a group was quite small in Experiment 1, which resulted in a relatively large effect size.

Furthermore, while we were unable to replicate the outcome of Experiment 1 in Experiment 2, and the converging evidence across the four experiments suggests there is very limited evidence for canine susceptibility to the Ponzo illusion, it remains possible that dogs are weakly susceptible to this illusion. Perhaps the dogs were originally susceptible to the illusion in Experiment 1 and then developed a dissociation between sensation and perception over time, as they underwent additional familiarization and testing trials. This could have led to more veridical responses. In a forthcoming paper we show that this same group of dogs are able to discriminate between two circles that differ in size by $20 \%$, with some able to discern differences of $15 \%$ and others differences of $10 \%$ (Byosiere et al., in prep). This may be 
instructive, as in the Ponzo illusion the two stimuli are physically identical, but appear slightly different to humans because of the illusory effect. In humans, this magnitude has been suggested to be about $18 \%$, however this is for line stimuli in a 'converging line' context (Chouinard, Unwin, Landry, \& Sperandio, 2016). Considering we used circle and square stimuli we cannot draw comparisons or make inferences about the degree of misperception in dogs. However, in a species not visually sensitive to small size differences, the illusion may be invisible, or very weak.

Additionally, it could be that illusion susceptibility in dogs may have been reduced in Experiments 2, 3, and 4 due to the repeated exposure to the illusory presentations. However, Parrish, Agrillo, Perdue, and Beran (2016) observed that both experimentally naïve and veteran capuchin monkeys performed similarly when presented with the Solitaire illusion (although with increased intra and inter individual variation than humans). It should be noted that the dogs were not rewarded for any particular choice in the test trials. Thus, perhaps in the absence of reinforcement, they were motivated to 'try out' different strategies and this prevented the results from being more coherent. An alternative explanation could be that of global precedence. Research suggests that dogs process stimuli in a globally oriented fashion, although there appears to be much individual variation (Pitteri, Mongillo, Carnier, \& Marinelli, 2014). It appears that individual global/local preferences are stable over time and there is an overall global bias observed in dogs (Mongillo, Pitteri, Sambugaro, Carnier, \& Marinelli, 2016). We suspect that susceptibility to geometrical illusions can be affected by preferential processing of local versus global aspects of a visual stimulus. While our familiarization processes were methodical, well controlled, and randomized, and we believed them necessary to introduce the dogs to the novel background images, it is possible that this extra training influenced the dogs to preferentially focus on local features, affecting their susceptibility to inducer cues in the global environment of the target object. However, additional research is needed to further assess this hypothesis. Studies of global/local precedence in dogs are largely based on performance in one precedence task (Navon, 1977), and it is clear from a number of experiments in humans that global processing should not be considered a singular construct, but rather an umbrella term to represent multiple independent mechanisms (Chouinard, Noulty, Sperandio, \& Landry, 2013; Chouinard et al., 2016).

Also of particular interest was the performance of specific individuals. Baxter consistently performed above $62 \%$ on the illusion trials across all four experiments, and this was significantly above chance in Experiments 2, 3, and 4. This may suggest individual variation in illusion susceptibility, as was observed in our previous study investigating susceptibility to the Ebbinghaus-Titchener illusion. Interestingly, Baxter is the only one of our subjects that we were able to successfully train to select the smaller stimulus. He is also extremely impulsive and confident in his choices, tending to choose quickly and decisively without pausing to consider options. We are currently training other dogs to select the smaller of two circles, and we are testing Baxter, as well as the other subjects on additional Ponzo stimuli.

Gabbie and Lulu performed significantly differently from chance in Experiment 3; however, their performance was in the opposite direction than observed in humans, demonstrating reversed illusion susceptibility. This reversed susceptibility was found in this same group of dogs when they were presented with the Ebbinghaus-Titchener illusion (Byosiere et al., 2016). Gabbie's performance was especially variable across Experiments, as she performed significantly different from chance, in Experiment 4, demonstrating illusion susceptibility in the same direction as humans. This may indicate that Gabbie and Lulu (in Experiment 3) were confused by the identically sized stimulus circles and used some aspect of the surrounding context to inform their choices, such that their errors were systematic but due to a confound rather than to illusion susceptibility. It is possible that they were demonstrating a preference for selecting the larger grid as opposed to the larger stimulus, although their performance in the familiarization trials showed that they were able to ignore the background image when the circles were different in size.

While these anomalies require further investigation, the results overall are particularly intriguing because they indicate that dogs may not be susceptible to the Ponzo illusion. To our knowledge, all animals previously tested have demonstrated susceptibility to this illusion in the same direction as humans (Feng et al., 2016). Therefore, if dogs are not susceptible, these findings have implications for 
underlying theoretical explanations. Recent research suggests that dogs may perceive illusory stimuli differently than humans (Byosiere et al., 2016; Miletto Petrazzini et al., 2016). These findings, together with the results of the current study, suggest that visual processing mechanisms may differ between humans, and dogs.

Clearly, additional research should be conducted to further assess Ponzo illusion susceptibility in dogs before more general conclusions are drawn. In order to maintain consistencies in facial morphology and, thus, ocular similarities, dogs of the same breed were used as participants in this study. They were also closely related and lived in the same environment, meaning that the results we observed may be breed-specific, specific to this genetic line, or the environmental context. In dogs, it appears that morphological diversity may be related to visual processing and eye structure (McGreevy, Grassi, \& Harman, 2003; Roberts, McGreevy, \& Valenzuela, 2010). McGreevy et al. (2003) observed that, while dog eye size is variable, it is strongly correlated with skull dimension, and that retinal ganglion cell distribution is highly variable and correlated with nose length. Considering the wide variety of morphological differences in dogs, future research should attempt to discern if breeds with flat faces (such as Boxers, Pugs, and Bulldogs) have different illusion susceptibility when compared to longer-nosed breeds (such as Labrador retrievers, German shepherds, and Border collies).

As discussed previously, additional investigation into whether training or testing methods influence the demonstration of direction of susceptibility to visual illusions is required, as is research establishing basic visual capabilities of dogs. Studies that find no apparent susceptibility often base that conclusion on the animal selecting the 'larger' or 'smaller' circle/line at chance levels. While this is a reasonable conclusion, other confounding factors (e.g., visual acuity, experimental stimuli) may be affecting these results. It would be instructive to see what happens when animals are taught to indicate a 'same size' option, in addition to the 'larger' or 'smaller' option on which they are normally trained. This is challenging from a training perspective, however, and is potentially limited to species capable of comprehending and demonstrating the abstract concept of same or different. We could find no studies testing this conceptual understanding in dogs.

Lastly, future research should extend this study to assess canine susceptibility to the Ponzo illusion in additional contexts. We were limited in the stimuli we could present, as the dogs were already well trained to select the larger (or smaller) of two circles presented in a horizontal plane on a computer monitor. This was assumed to be satisfactory because the illusions were robust when viewed by the researchers. However, future research should examine Ponzo illusion susceptibility using a presentation with vertical alignment, which may provide stronger linear perceptive cues, in both a classical and pictorial form. Such stimuli would more closely approximate those used with rhesus macaques (Fujita, 1996), horses (Timney \& Keil, 1996), and rats (Nakagawa, 2002).

To the best of our knowledge, this study is the first to examine canine susceptibility to the Ponzo illusion. Recent research in dogs suggests that they may perceive certain illusions in an opposite manner than humans and most other animal species (Byosiere et al., 2016), or may not demonstrate susceptibility at all (Byosiere et al., 2016; Miletto Petrazzini et al., 2016). Across four presentations of the Ponzo illusion, we could not find evidence for discernible susceptibility to the Ponzo illusion in a two-choice discrimination paradigm. In Experiment 1, susceptibility to the Ponzo illusion was observed at the group level; however, no individual dog performed significantly above chance. In Experiments 2, 3, and 4, no significant results were observed at the group level, although individual dogs did show some evidence of susceptibility. This was sometimes in the same direction as humans but, at other times, was in the reverse direction. These findings require replication but potentially have implications for theoretical explanations of the Ponzo illusion. Several other animal species have demonstrated susceptibility to the Ponzo illusion in the same direction as humans, but we could find no other reports of any animal not being susceptible. Considering that susceptibility to the Ponzo illusion has been suggested to require high levels of visual processing in humans, this divergence in findings may suggest that the mechanisms underlying perception of the Ponzo stimuli differ across species, resulting in only some experiencing the illusion. 


\section{Acknowledgements}

We are grateful to Eva Worden for her help during the training process, Joyce Wuister and Diana Rayment for their help during the data collection process, Ron Wheeler for his assistance in the construction and design of our apparatus, and Davis Vorva for his expertise in program design. This research was carried out with the support of La Trobe University Postgraduate Research Scholarships and La Trobe University Full Fee Research Scholarships.

Conflict of Interest: The authors declare that they have no conflict of interest.

\section{References}

Agrillo, C., Parrish, A. E., \& Beran, M. J. (2014). Do primates see the solitaire illusion differently? A comparative assessment of humans (Homo sapiens), chimpanzees (Pan troglodytes), rhesus monkeys (Macaca mulatta), and capuchin monkeys (Cebus apella). Journal of Comparative Psychology, 128, $402-413$.

Barbet, I., \& Fagot, J. (2002). Perception of the corridor illusion by baboons (Papio papio). Behavioural Brain Research, 132, $111-115$.

Bayne, K. A. L., \& Davis, R. T. (1983). Susceptibility of rhesus monkeys (Macaca mulatta) to the Ponzo illusion. Bulletin of the Psychonomic Society, 21, 476 - 478.

Brislin, R. W., \& Keating, C. F. (1976). Cultural differences in the perception of a three-dimensional Ponzo illusion. Journal of Cross-Cultural Psychology, 7, 397 - 412.

Byosiere, S.-E., Feng, L. C., Woodhead, J. K., Rutter, N. J., Chouinard, P. A., Howell, T. J., \& Bennett, P. C. (2016). Visual perception in domestic dogs: Susceptibility to the Ebbinghaus-Titchener and Delboeuf illusions. Animal Cognition, 20, $435-448$.

Chouinard, P. A., Noulty, W. A., Sperandio, I., \& Landry, O. (2013). Global processing during the Müller-Lyer illusion is distinctively affected by the degree of autistic traits in the typical population. Experimental Brain Research, 230, $219-231$.

Chouinard, P. A., Unwin, K. L., Landry, O., \& Sperandio, I. (2016). Susceptibility to optical illusions varies as a function of the autism-spectrum quotient but not in ways predicted by local-global biases. Journal of Autism and Developmental Disorders, 46, 2224 - 2239.

Feng, L. C., Chouinard, P. A., Howell, T. J., \& Bennett, P. C. (2016). Why do animals differ in their susceptibility to geometrical illusions? Psychonomic Bulletin \& Review, 24, 262 - 276.

Fujita, K. (1996). Linear perspective and the Ponzo illusion: A comparison between rhesus monkeys and humans. Japanese Psychological Research, 38, 136 - 145.

Fujita, K. (1997). Perception of the Ponzo illusion by rhesus monkeys, chimpanzees, and humans: Similarity and difference in the three primate species. Perception \& Psychophysics, 59, $284-292$.

Fujita, K., Blough, D. S., \& Blough, P. M. (1991). Pigeons see the Ponzo illusion. Animal Learning \& Behavior, 19, $283-293$.

Geisler, W. S., \& Diehl, R. L. (2002). Bayesian natural selection and the evolution of perceptual systems. Philosophical Transactions of the Royal Society B, 357, 419- 448.

Gellermann, L. W. (1933). Chance orders of alternating stimuli in visual discrimination experiments. The Pedagogical Seminary and Journal of Genetic Psychology, 42, 206-208.

Gregory, R. L. (1963). Distortion of visual space as inappropriate constancy scaling. Nature, 199, $678-680$.

Gregory, R. L. (2015). Eye and brain: The psychology of seeing. Princeton, NJ: Princeton University Press.

Haber, R. N., \& Hershenson, M. (1973). The psychology of visual perception. Oxford, UK: Holt, Rinehart, \& Winston.

Humphrey, N. K., \& Morgan, M. J. (1965). Constancy and the geometric illusions. Nature, 206, $744-745$.

Imura, T., Tomonaga, M., \& Yagi, A. (2008). The effects of linear perspective on relative size discrimination in chimpanzees (Pan troglodytes) and humans (Homo sapiens). Behavioural Processes, 77, 306 - 312.

Jacobs, G. H., Deegan, J. F., Crognale, M. A., \& Fenwick, J. A. (1993). Photopigments of dogs and foxes and their implications for canid vision. Visual Neuroscience, 10, $173-180$.

Kasparson, A. A., Badridze, J., \& Maximov, V. V. (2013). Colour cues proved to be more informative for dogs than brightness. Proceedings of the Royal Society B: Biological Sciences, 280, 20131356.

Lazareva, O. F., Miner, M., Wasserman, E. A., \& Young, M. E. (2008). Multiple-pair training enhances transposition in pigeons. Learning \& Behavior, 36, $174-187$. 
Lazareva, O. F., Wasserman, E. A., \& Young, M. E. (2005). Transposition in pigeons: Reassessing Spence (1937) with multiple discrimination training. Animal Learning \& Behavior, 33, $22-46$.

Lazareva, O. F., Young, M. E., \& Wasserman, E. A. (2014). A three-component model of relational responding in the transposition paradigm. Journal of Experimental Psychology: Animal Learning and Cognition, 40, 63 80 .

McGreevy, P., Grassi, T. D., \& Harman, A. M. (2003). A strong correlation exists between the distribution of retinal ganglion cells and nose length in the dog. Brain, Behavior and Evolution, 63, $13-22$.

Miletto Petrazzini, M. E., Bisazza, A., \& Agrillo, C. (2016). Do domestic dogs (Canis lupus familiaris) perceive the Delboeuf illusion? Animal Cognition, 20, 427 - 434.

Miller, P. E., \& Murphy, C. J. (1995). Vision in dogs. Journal-American Veterinary Medical Association, 207, 1623 -1634 .

Mongillo, P., Pitteri, E., Sambugaro, P., Carnier, P., \& Marinelli, L. (2016). Global bias reliability in dogs (Canis familiaris). Animal Cognition, 20, $257-265$.

Murayama, T., Usui, A., Takeda, E., Kato, K., \& Maejima, K. (2012). Relative size discrimination and perception of the Ebbinghaus illusion in a bottlenose dolphin (Tursiops truncatus). Aquatic Mammals, 38, 333 - 342.

Nakagawa, E. (2002). Rats respond to configurations of stimuli. The Psychological Record, 52, $531-558$.

Nakamura, N., Watanabe, S., \& Fujita, K. (2008). Pigeons perceive the Ebbinghaus-Titchener circles as an assimilation illusion. Journal of Experimental Psychology: Animal Behavior Processes, 34, 375 - 387.

Nakamura, N., Watanabe, S., \& Fujita, K. (2014). A reversed Ebbinghaus-Titchener illusion in bantams (Gallus gallus domesticus). Animal Cognition, 17, $471-481$.

Navon, D. (1977). Forest before trees: The precedence of global features in visual perception. Cognitive Psychology, $9,353-383$.

Neitz, J., Geist, T., \& Jacobs, G. H. (1989). Color vision in the dog. Visual neuroscience, 3, $119-125$.

Newman, C. V., \& Newman, B. M. (1974). The Ponzo illusion in pictures with and without suggested depth. The American Journal of Psychology, 87, 511 - 516.

Parrish, A. E., Agrillo, C., Perdue, B. M., \& Beran, M. J. (2016). The elusive illusion: Do children (Homo sapiens) and capuchin monkeys (Cebus apella) see the Solitaire illusion? Journal of Experimental Child Psychology, 142, $83-95$.

Pitteri, E., Mongillo, P., Carnier, P., \& Marinelli, L. (2014). Hierarchical stimulus processing by dogs (Canis familiaris). Animal Cognition, 17, $869-877$.

Pressey, A. (1971). An extension of assimilation theory to illusions of size, area, and direction. Perception \& Psychophysics, 9, $172-176$.

Roberts, T., McGreevy, P., \& Valenzuela, M. (2010). Human induced rotation and reorganization of the brain of domestic dogs. PLoS One, 5, e11946.

Salva, O. R., Rugani, R., Cavazzana, A., Regolin, L., \& Vallortigara, G. (2013). Perception of the Ebbinghaus illusion in four-day-old domestic chicks (Gallus gallus). Animal Cognition, 16, 895 - 906.

Segall, M. H., Campbell, D. T., \& Herskovits, M. J. (1963). Cultural differences in the perception of geometric illusions. Science, 139, $769-771$.

Song, C., Schwarzkopf, D. S., \& Rees, G. (2011). Interocular induction of illusory size perception. BMC Neuroscience, 12, 27.

Sovrano, V. A., Albertazzi, L., \& Salva, O. R. (2014). The Ebbinghaus illusion in a fish (Xenotoca eiseni). Animal Cognition, 18, $533-542$.

Sperandio, I., \& Chouinard, P. A. (2015). The mechanisms of size constancy. Multisensory Research, 28, 253-283.

Tapp, P. D., Siwak, C. T., Head, E., Cotman, C. W., Murphey, H., Muggenburg, B. A., ...Milgram, N. W. (2004). Concept abstraction in the aging dog: Development of a protocol using successive discrimination and size concept tasks. Behavioural Brain Research, 153, $199-210$.

Timney, B., \& Keil, K. (1996). Horses are sensitive to pictorial depth cues. Perception, 25, 1121 - 1128.

Wang, G.-d., Zhai, W., Yang, H.-c., Fan, R.-X., Cao, X., Zhong, L., ...Cheng, L.-g. (2013). The genomics of selection in dogs and the parallel evolution between dogs and humans. Nature Communications, 4, 1860.

Watanabe, S., Nakamura, N., \& Fujita, K. (2011). Pigeons perceive a reversed Zöllner illusion. Cognition, 119, 137 -141 .

Watanabe, S., Nakamura, N., \& Fujita, K. (2013). Bantams (Gallus gallus domesticus) also perceive a reversed Zöllner illusion. Animal Cognition, 16, 109 - 115. 